\title{
A Poor Concept Script
}

\author{
Hartley Slater \\ Department of Philosophy \\ University of Western Australia \\ slaterbh@cyllene.uwa.edu.au
}

Received by Greg Restall

Published August 2, 2004

http://www .philosophy .unimelb.edu.au/ajl/2004

(C) 2004 Hartley Slater

\begin{abstract}
The formal structure of Frege's 'concept script' has been widely adopted in logic text books since his time, even though its rather elaborate symbols have been abandoned for more convenient ones. But there are major difficulties with its formalisation of pronouns, predicates, and propositions, which infect the whole of the tradition which has followed Frege. It is shown first in this paper that these difficulties are what has led to many of the most notable paradoxes associated with this tradition; the paper then goes on to indicate the lines on which formal logic — and also the lambda calculus and set theory-needs to be restructured, to remove the difficulties.

Throughout the study of what have come to be known as first-, second-, and higher-order languages, what has been primarily overlooked is that these languages are abstractions. Many well known paradoxes, we shall see, arose because of the elementary level of simplification which has been involved in the abstract languages studied. Straightforward resolutions of the paradoxes immediately appear merely through attention to languages of greater sophistication, notably natural language, of course. The basic problem has been exclusive attention to a theory in place of what it is a theory of, leading to a focus on mathematical manipulation, which 'brackets off' any natural language reading.
\end{abstract}

I first remind readers that reflexive and personal pronouns only arise in context sensitive languages, and so do not have a direct representation in the context insensitive language of recent formal logic. That leads to resolutions of Russell's Paradox, the Paradox of Heterologicality, and related antinomies.

Fregean predicate logic has no representation of reflexive pronouns, as such, nor therefore of any predicate in which they occur. Instead it has tried to make do by relying on such equivalences as that between ' $x$ is not a member 
of itself' and ' $x$ is not a member of $x$ ', taking the whole of the latter as a 'predicate', whereas it is merely the form of a sentence. Like 'is not a member of itself' in the former, 'is not a member of $x$ ' is the predicate in the latter, even though it contains a variable. The confusion between forms of sentences and predicates goes along with a confusion between facts and properties, which are commonly distinguished in natural languages, but which have come to be conflated in lambda abstraction and set abstraction languages. Isn't the property in question

$$
\lambda x(x \text { is not a member of } x)
$$

which is a constant? No: the predicate is 'is not a member of itself', so it is the functional

$$
\lambda x(x \text { is not a member of } s)
$$

where ' $s$ ' is a pronoun for the immediate subject, and not the constant term ' $\lambda x(x$ is not a member of $x)$ ' that expresses the property. It is not a property but a fact if $x$ is a not a member of $x$.

Certainly those things with the property in question are those things for whom the same form of fact holds, i.e.

$$
(\forall x)(x \text { is not a member of itself } \equiv x \text { is not a member of } x) \text {. }
$$

But that does not mean that each of those things has the same constant property, i. e. that the property in question is not functional. Indeed, the equivalence itself is what gives the form of the function in this case. So one merely gets ' $x$ is not a member of itself' equivalent to ' $x$ is a member of its complement' $(x \in\{y \mid y \notin x\})$, or ' $x$ is one of the sets it is not a member of' $(x \in\{y \mid x \notin y\})$, not ' $x$ is a member of $R$ ' for some fixed set $R$. There is a set spoken of, but it is a variable one dependent on something else, i. e. $(\forall x)(\exists y)(x \in y \equiv x \notin x)$, not $(\exists y)(x)(x \in y \equiv x \notin x)$, and there is no contradiction obtainable from $(\forall x)(x \in\{y \mid y \notin x\} \equiv x \notin x)$, or $(\forall x)(x \in\{y \mid x \notin y\} \equiv x \notin x)$.

A predicate is a part of a sentence, not the form of one; and it is then complemented by the subject. So what was missing in Fregean abstracts was the identification of a subject place where the variable is bound. Without a subject place being nominated in a sentence there is no way to isolate the remaining part which is the predicate; and in sentences with multiple possible subjects, therefore, there will be several possible predicates associated with them: they are commonly distinguished by their voices, i. e. by whether they are active or passive. 'John shaves John' involves not the single 'property' $\lambda x(x$ shaves $x)$, but the property of shaving John $(\lambda x(x$ shaves John $))$, if the first 'John' is taken as subject, and the property of being shaved by John $(\lambda x$ (John shaves $x))$, if the second 'John' is taken as subject. In 'John shaves himself' the predicate is 'shaves himself', but in this case the men who shave themselves do not thereby share a constant property, since this predicate is context sensitive, and so it does not collect up a set of things with a constant property. If each of $A, B$ 
and $C$ shave $C$, they do the same thing - shave $C$ - but if each of $A, B$, and $C$ shaves himself, or say, in a ring, shaves his neighbour on his left, then they only do the same kind of thing, i. e. what they do merely has a common functional expression-shave $f(s)$, where $s$ is the subject. Of course, those men who shave themselves might still be listed, but being a member of the set $\{A, B, C\}$ clearly does not identify the men in question either as self-shavers, or as leftneighbour-shavers. We shall see, at the end of this paper, how to formulate an embracing set in connection with all the sets which are not members of themselves, but that again, of course, cannot be a set of those non-self-membered sets, on pain of paradox.

The need to consider functional predicates re-appears with respect to the paradox of Heterologicality. For well known contradictions are produced when the reflexive pronoun in 'is not applicable to itself' is taken not to have a variable reference, so that the whole is read as having a fixed sense: then ' $x$ is not applicable to itself' seemingly comes out equivalent to ' $x$ is $h$ '. But the resultant confusion is eliminated when one allows the pronoun to have its referent fixed through its association with the local subject term. In the sense, if not the syntax of ' $x$ is not self-applicable if and only if $x$ is not applicable to $x$ ' there are four occurrences of ' $x$ ' and not just three, and so the predicate on the left is not replaceable by any constant one. And if one places 'is not self-applicable' at each of these four places there is no paradox. Replacing it at just the three places where there is an ' $x$ ' produces the notoriously puzzling contradiction: 'is not self-applicable' is not self-applicable if and only if 'is not self-applicable' is not applicable to 'is not self-applicable'. But replacing it in all four places produces: 'is not self-applicable' is not 'is not self-applicable'-applicable if and only if 'is not self-applicable' is not applicable to 'is not self-applicable'-which is paradox free.

Likewise in Quine's famous reformulation 'yields a falsehood when appended to its own quotation': here 'its own' has a reference dependent on the subject to which the predicate is attached, and so the predicate is only properly expressible in a context sensitive language. In "'yields a falsehood when appended to its own quotation' yields a falsehood when appended to its own quotation" the second 'its own' has a reference determinable from the context, but the first 'its own' has no determinate reference at all, since it has been abstracted from any context by merely being part of a mentioned expression. As a result, in the required full sense, that mentioned expression, without a determinate reference for the pronoun, can never get appended to its own quotation, since when appended it is not mentioned but used, and thereby a portion of it necessarily has a different sense from when it is not appended, through the context sensitivity, which gives a determinate reference to the pronoun in it.

We next see how Frege's formalisation of elementary predicates has led to problems with 'The Unity of the Proposition', and 'The concept horse', and contrib- 
uted to the Paradox of Predication and problems with an objectival reading of second-order quantifiers.

For the difficulty with seeing all of the above compounds if one is attached to Frege's ' $P x$ ' symbolisation of elementary sentences. If ' $P$ ' there stands for a property or concept, and one abstracts ' $x$ ' from ' $P x$ ', then what is left but a referring phrase to that property or concept? This form of symbolisation arose because of Frege's assimilation of concepts to functions. Concepts were not objects, he believed, making 'the concept horse', which Frege agreed was a referring phrase to an object, paradoxically not a referring phrase to a concept. Instead, concepts, like ' $P$ ', were functions, and with the subject $x$ taken as the argument of the function, the value of the whole subject-predicate expression was supposedly a truth value, in parallel with ' $f x=y$ '. But this construal of truth as the value of a function is erroneous, as we shall see. More directly, if we wrote for the form of an elementary predication, not 'P $x$ ', but ' $x$ has P', where ' $P$ ' is a referring phrase to the property or concept, it would be more clear that abstracting the subject leaves not a referring phrase to that property or concept, but merely the predicate in question, 'has P'. In fact, in the symbolism ' $\mathrm{Px}$ ' the ' $\mathrm{P}$ ' is doing double duty for a predicative expression involving a finite form of the verb, like 'is a horse', along with a referring phrase to the property alone, like 'being a horse', which would allow objectival second-order quantification. But, of course, on the latter reading of ' $P x$ ', ' $x$, the property P' is not a coherent sentence, giving rise to the problem of how a proposition is unified, and is not just a list of names.

It characterises the 'bracketed' approach to Fregean Logic which is more prevalent, that writers on the unity of the proposition, and Frege's problem with the concept borse, for instance, do not commonly remember in their own practise the difference between 'is a horse', and its nominalisation 'being a horse', so that the absence of predicate nominalisations in Frege's symbolism is not brought up as an evident difficulty with it (c. f. Slater 2000). There is no lack of unity in 'Dobbin is a horse' like there is in 'Dobbin, being a horse', and the predicate in the former clearly does not refer to any concept, since it is not a referring expression at all, not being nominalised, as in the latter. As we shall see, it is a predicate like 'is a horse' which is functional, needing a subject term to be inserted to form a complete thought, and that leaves the associated concept phrase 'being a horse' to be unproblematically the designator of an (abstract) object.

That, of course, relieves us of one difficulty with the Paradox of Predication: ' $(\exists P)(x=P \wedge \neg P x)$ ' is ungrammatical. The objectival quantification ' $(\exists P)(x=P \wedge \neg(x$ has $P))$ ', in which 'P' is a referring phrase to a property, is not ungrammatical, by contrast. Alternatively (c. f. Kneale and Kneale i962, page 602), if one introduces the nominaliser ' $\$$ ', so that ' $\$ y P y$ ' refers to the property while ' $P$ ' remains descriptive (and so equivalent to 'has $\$ y P y$ '), that would allow a further expression, ' $(\exists P)(x=\$ y P y \wedge \neg P x)$ ', to be well formed, in which the quantification is substitutional, with ' $P$ ' then an open predicate. 
In either case, however, we would have the question, as with Heterologicality, and Russell's Paradox, whether the resulting predicate expressed a single, unambiguous property. Indeed, if $(\exists P)(x=\S y P y \wedge \neg P x) \equiv Q x$, then there would be a contradiction when $x=\$ y Q y$.

That contradiction proves indirectly that there is no single property, but what is the direct proof of this? How is it that ' $(\exists P)(a=P \wedge \neg(a$ has $P))$ ', and ' $(\exists P)(b=P \wedge \neg(b$ has $P))$ ', do not say $a$ and $b$ have the same property? The matter is now plain. Certainly these may seem to say the same thing about $a$ as about $b$, but the sameness is only in the linguistic expression, which is systematically ambiguous. The common predicate 'is a property which it does not possess' contains a pronoun which refers back to the given subject term, so while the same syntactic item is involved, it is context sensitive with respect to its referent, which means that the whole is replaceable by 'is a property which a does not possess' and 'is a property which b does not possess', respectively, in the two cases. Geach once considered this kind of possibility, in connection with Heterologicality, but he thought one could remove the variability in other re-formulations (Geach 1972, 90). Certainly one can move the context sensitive element in 'is a property which it does not possess' to another place, as in 'is a property, but does not possess that property', or 'is, but does not possess the same property', but the referent of 'that property', and 'the same property' is variable as before.

Some of the above confusions in Frege's 'concept script' are replicated at the level of sentences. Frege said (Geach and Black 1952, 64):

One might be tempted to regard the relation of the thought to the True not as that of sense to reference, but rather as that of subject to predicate. One can indeed say: 'The thought, that 5 is a prime number, is true.' But closer examination shows that nothing more has been said than in the simple sentence ' 5 is a prime number.' The truth claim arises in each case from the form of the declarative sentence, and when the latter lacks its usual force, e.g. in the mouth of an actor upon the stage, even the sentence 'The thought that 5 is a prime number is true' contains only a thought, and indeed the same thought as the simple ' 5 is a prime number.' It follows that the relation of the thought to the True may not be compared with that of subject and predicate. Subject and predicate (understood in the logical sense) are indeed elements of thought; they stand on the same level for knowledge. By combining subject and predicate, one reaches only a thought, never passes from sense to reference, never from a thought to its truth value. One moves at the same level but never advances from one level to the next. A truth value 
cannot be part of a thought, any more than, say, the Sun can, for it is not a sense but an object.

If our supposition that the reference of a sentence is a truth value is correct...

But it is painfully evident, to anyone who does not bracket off their own practise, that Frege's supposition is not correct, since sentences, unlike their nominalisations, simply are not referring expressions. Moreover, as Frege himself admits in this passage, the referent of 'that 5 is a prime number' is a thought, and it is that sort of thing which can be said to have a truth value. Frege did have an expression for such thoughts-with a horizontal line replacing 'that'-but regrettably they were not taken as subject terms, allowing him no natural expression not only for modal remarks like 'that $p$ is necessary', but also more general comments of the same kind, like 'that $p$ is obvious', 'that $p$ is surprising' etc. With thoughts allowed a subject place in this way it becomes clear that truth is a property of thoughts, and it is the sentence nominalisation 'five's being a prime number' which refers to its being true that 5 is a prime number. Frege's judgment stroke, which was put before selected thoughts, is possibly best seen as performing this sentence nominalising function, with the consequent marking of something's being true, since certainly, as the passage above shows, Frege did not take it to mean the predicative 'it is true', or even 'it is asserted'.

Is the concept of being a prime number, then, a function which takes the value True when applied to the argument 5 ? No: the concept of being a prime number, like the concept of being a horse, is an object (an abstract object), since predicate nominalisations are straightforwardly referential. A concept is thus 'saturated' and so cannot be a function; it is the associated predicate, 'is a prime', or 'is a horse', which is incomplete, and incomplete expressly because it is merely a part of a sentence. Thus we may complete such a sentence by inserting ' 5 ' or 'Dobbin' in the remaining gap, but when asserting the whole we then do not transform 5, or Dobbin into the True, we merely express a thought, which has truth as one of its properties. In the mouth of an actor on a stage certainly the same thought might be entertained rather than asserted, but that is just a matter of the associated sentence being quoted, and so being merely mentioned, not used. Use requires no assertion sign, merely the dropping of quotes, and the use of a sentence rather than the quoting of it is crucially what is involved in truth assessments like 'that $p$ is true'.

The nominaliser 'that' has been symbolised ' $\$$ ' more recently (c. f. Kneale I972, Haack 1978, Chapter 6). By prefacing a used sentence ' $p$ ', which expresses a thought, it makes ' $\$ p$ ' into a denoting phrase which refers to the thought. The referential combination ' $\$ p$ ' is closely related to, although distinct from a plain sentence nominaliser: Tarski's being wrong, for instance, is the same as its being true that Tarski is wrong, because of the propositional truth scheme ' $p \equiv$ it is true that $p$ '.

There are still many, of course, who accept Tarski's sentential T-scheme, 
thinking truth is primarily expressed by a predicate of sentences, instead of a predicate of 'that' clauses (c. f. Prior 1971, Chapter 7). The tradition at this point has departed from Frege, in accepting that 'is true' is a straight predicate, but has still held onto the Fregean dismissal of 'that p' as a possible subject term. But in selecting, instead, mentioned sentences as the subject of judgments of truth it has run into a host of troubles. For, notoriously, a paradoxical form of self-reference is obtained in connection with identities like " $t=$ ' $t$ is not true'", if truth is a disquotational property of sentences. Then ' $t$ is not true' is true if and only if $t$ is true (on account of the identity), but also true if and only if $t$ is not true (by disquotation). Revisiting the points made above, about Quine's reformulation of the heterologicality predicate, helps one to see where the fallacy lies in this disquotational argument, however. For in such an identity as " $t=$ ' $t$ is not true'", while the referent of the first ' $t$ ' is thereby given, there is no referent provided for the second ' $t$ ', since it is merely mentioned. So there is crucially no implication that $(\exists x)(x=$ ' $x$ is not true'), which means there is no real self reference-the relation is merely a nominal one between $t$ and something which includes the letter ' $t$ ' (c. f. Slater 2002). Hence when disquoting the ' $t$ is not true' above to obtain the implication that $t$ (sic) is not true, one is illicitly assuming a reference for an item lacking a reference, and thereby producing a transposition, from mention to use, as invalid as that which would be involved in quantifying over a quoted place.

By contrast, not even the appearance of a contradiction is obtainable if truth is primarily expressed by an operator on sentences, i. e. a predicate of 'that' clauses, making truth a property of thoughts. For there is then no opportunity for paradoxical substitution of the above identity: one says that $t$ is not true, since sentences are no longer the bearers of truth, and that simply means that ' $t$ is not true' is not true. In saying that $t$ is not true, isn't one asserting that the sentence one then uses (' $t$ is not true') is true? No: one is not saying ' $p$ ' is true when one says it is true that $p$, because use is not mention, and operators are not predicates, and so, a fortiori, not meta-linguistic predicates. The sentence ' $t$ is not true' is not said to be true when one says that $t$ is not true, since one is then saying, instead, that the referent of the 'that' clause is true. There is no self-reference available with operator constructions, of course: mereology prevents 'it is not true that $p$ ' from itself being ' $p$ ', since a whole cannot contain itself as a proper part. So there is no way that appropriate self-reference can arise, if the subject of the judgment of truth is of the form ' $\$ p$ '.

Can't we get self-reference, though, using other subjects in such judgments? What if Tarski utters at time $t$ just 'What Tarski states at time $t$ is not true'? Then, it seems, what Tarski states at time $t$ is that what Tarski states at time $t$ is not true, and we have a characteristic impasse. But can we be so positive about what Tarski states at the given time? It is here we must remember the possibility that the language is context sensitive, with the consequent distinction between statements and propositions being required to identify the bearers of truth (c. f. Haack 1978, Chapter 6). Reading the statement made by 
the use of a sentence off the face of that sentence is not guaranteed if the language is context sensitive-only the proposition expressed is obtainable that way. And what makes the given sentence context sensitive (and similarly for other like sentences) is that what its subject term refers to depends on who utters it (or when, or where it is uttered). If someone else utters it, for instance, and Tarski says something else at time $t$, then a different statement is made from any statement made when Tarski utters it at time $t$. And that latter case is most like the case where someone else utters it, and Tarski says nothing at all at time $t$, since if he states no single thing with his words, then there is no way to identify what he states, and so there is no paradox.

Writing 'what Tarski states' as ' $E r S t r$ ', certainly then Tarski states that what he states is not true, i. e. St $\$ \neg T \varepsilon r S t r$, but only supposing further that only one statement is involved can one obtain a contradiction. For St $\$ \neg T \varepsilon r S t r$ entails $(\exists r) S t r$, i. e. that there is something Tarski states, so we have SterStr, i. e. that Tarski states what he states, by the epsilon definition of the quantifier- $-(\exists r) \mathrm{Pr}$ is equivalent to $\operatorname{P\varepsilon rPr}$ (see Leisenring 1969). But one needs the further assumption that $(\exists$ !r)Str to get $(\forall s)$ (Sts $\supset s=\S \neg T \varepsilon r S t r)$, and only on this basis does it follow that $\varepsilon r S t r=\$ \neg T \varepsilon r S t r$, i. e. that what Tarski states is that what Tarski states is not true. This identity then gives TerStr $\equiv \mathrm{T} \S \neg \mathrm{T} \varepsilon r S t r$, and so $\mathrm{T} \varepsilon r S t r \equiv \neg T_{\varepsilon} r S t r$, which is the contradiction; but all that follows is that the further assumption is false, i. e. that not just one statement is involved. As in the previous paradoxes with predicates, the paradox with Tarski's sentence (and the like) is resolved through recognising it is ambiguous: we have SterStr

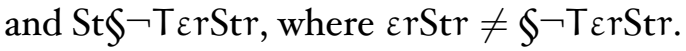

IV

But if the above demonstrates that many classic paradoxes arose because of the level of abstraction in the languages studied, the question subsequently arises of what form a more sophisticated formal logic might take. Maybe it is clear that attention to the context sensitivity of pronouns clears up many classic paradoxes, attention to predicate nominalizations clears up other notorious difficulties, and attention to sentence nominalizations yet others, even so, it may be asked, what is the general form, and, in particular, the syntax and semantics of any reformed logical symbolism to be? The main problem here is accepting that it must be radically different in certain respects, even if in others the significant differences are fairly minor. But some of the major changes merely take the form of surgery on what is old, and do not add much which is new, so the final result is not too strange. Thus, for a start, there is no pressing requirement to add pronouns to the symbolism, since pronouns are an accidental element in language, and in any context of use are replaceable with the nouns they abbreviate.

One minor, but still significant change occurs in the case of propositions and operators on them, like 'it is necessary that p', 'it is believed that p', and so 
on. The study of such propositional operators has developed since the i960s without access to a generally accepted expression for the equivalent, non-cleft forms 'that $p$ is necessary', 'that $p$ is believed' etc., and clearly the inability to formalise such equivalences has arisen because of the deliberate absence in the heritage from Frege of subject terms of the kind 'that p'. Thus what has been written, in modal logic, 'Lp', with 'L' a propositional operator, could be written ' $N \$ p$ ', with ' $N$ ' a predicate, making ' $N \S$ ' merely a more structured form of ' $L$ '. But without ' $\$$ ', or the like, in the symbolism no such equivalence can be expressed. Prior, who promoted at great length the study of propositional operators, in his posthumous book 'Objects of Thought', did so without the advantage of Kneale's formalisation of 'that', and this led directly to the well-known difficulties with Prior's treatment of propositional quantification, notably the difficulty of giving an objectival reading of his propositional quantifiers (Slater 200I). Moreover, Prior also missed the fact that, with the more articulated grammar for modal and general intensional constructions, their logic becomes directly a form of first-order predicate logic, with the appropriate subject and predicate terms-although this is best extended by adding propositional epsilon terms, as in the example above, in parallel with the common conservative extension of the predicate calculus to the epsilon calculus. Epsilon terms are particularly valuable in formalising the descriptive replacements for certain anaphoric pronouns, as I have shown in a good number of articles starting from Slater 1986. Thus the 'it' in 'Tarski stated something. It was that what he stated was false' has the replacement 'what Tarski stated', so that the whole becomes ' $(\exists r)$ Str $\wedge \varepsilon r S t r=\$ \neg T \varepsilon r S t r$ '.

Evidently there are more basic mental pre-occupations which might need to be overcome, before allowing into logic referential terms to thoughtsnotably Quinean fears of 'intensional entities' like propositions, and Quinean quotational theories of belief, for instance, which involve the same fear, and try to start from properties of sentences, instead. But the latter pre-occupation, as with Tarskian quotational theories of truth, ignores the difference between two senses of 'say', namely 'utter', and 'state' (c. f. above, and Kneale 1972, page 230); and the former ignores related aspects of 'that' clauses. We say, for instance, 'That John was already there was surprising', and so predicate something of a subject of the form 'that $\mathrm{p}$ ', but the nominaliser 'that' is then clearly not a quotation maker, since what was surprising was not the sentence 'John was already there', but instead the fact that John was already there.

This leads us to the most radical change, which involves a fundamental reassessment of what semantics is about; indeed, in one sense, it becomes quite inappropriate to ask for a semantics of the more sophisticated language. For meta-linguistic forms like ' $p$ ' is true', in terms of which semantics traditionally has been expressed, entirely lose the significance they had. Are not such forms needed to give us an understanding of the object language? Not if that language is our Mother Tongue, and understanding is obtained just through use. Prior saw that, because of this move to use, the move to the operator approach spelt 
the end of Tarskian semantics (c. f. Prior 1971, Chapter 7), but that does not mean it is the end of formal semantics entirely. For when using the propositional operator form 'it is true that p', instead of the meta-linguistic form ' $p$ ' is true', a truth locution is still involved, only now in the 'object' language itself. But that means it has to be fully appreciated there is no problem with that, i. e. that a language can be semantically closed without contradiction, since the tradition from Tarski has come firmly to believe that a language cannot be semantically closed without being defeated by The Liar Paradox. But, in addition to the above resolution of this paradox, the consistency of the epsilon calculus is readily shown (Leisenring I969, p48), and Goodstein proved the consistency of his operator theory in the first formal paper on the subject (Goodstein I958).

In addition to this consistency, the now propositional T-scheme ' $\mathrm{T} \$ p \equiv p$ ' means, for instance, that the Principle of Bivalence (it is true that $p$ or it is true that $\neg p$ ) is logically equivalent to the Law of the Excluded Middle ( $p \vee \neg p$ ), and lines in a truth table such as 'If it is true that $p$ and it is not true that $q$, then it is not true that $p \wedge q$ ' are equivalent to propositional theses like ' ( $p \wedge \neg q) \supset \neg(p \wedge q)$ '. So the move to a Priorian semantics not only produces a semantically closed language but also collapses, amongst other things, the usual soundness and completeness proofs in propositional logic.

The grammar of second order logic needs to be revised, of course, once nominalised predicates are incorporated. But in fact this has already has been done, to the required extent, by Cocchiarella (Cocchiarella I986, Chapters IV, $\mathrm{V}, \mathrm{VI})$. Thus Cocchiarella writes ' $[\lambda \times \varphi(\mathrm{x})](\mathrm{)}$ ' as a complex predicate, when $\varphi(x)$ is a wff, with ' $[\lambda \times \varphi(x)]$ ' as the corresponding singular term (Cocchiarella I987, p. I6I). These lambda expressions are in place of the everyday 'has P' and ' $P$ ', with 'P' a referring phrase, and Kneale's ' $P$ ' and ' $\$ y P y$ ', with ' $P$ ' an open predicate, mentioned before. But Cocchiarella did not point out the consequent relief provided for the problem of the unity of the proposition, and the problem about the concept horse, or the problem about concepts being functions, and the resultant problem about truth values being the referents of sentences. Cocchiarella was merely concerned with Russell's Paradox and the Paradox of Predication, in the application of his enriched language. As we have seen, however, the basic problem with the latter two paradoxes lies elsewhere, in problems about the formalisation of pronouns.

The resolution of these problems about pronouns principally impacts on the allowable abstracts in the Lambda Calculus, and Set Theory, showing there are wider repercussions of this matter than those just in Formal Logic. Take the case of 'John shaves John' which we looked at before. Since abstracting just the first 'John', or the second 'John' from this sentence leaves a predicate still involving 'John', it certainly follows that we must abstract both 'John's to obtain a predicate which lacks 'John' entirely. But is that predicate then still just a predicate of 'John'? It is with respect to the subject of this predicate that the traditional Lambda Calculus, and Set Theory misconstrued the grammar of the matter, and thereby the possible subject-predicate divisions of 'John shaves 
John'. For if one wants a predicate that lacks 'John' entirely, one must talk not about some property of fohn, but a property of the subject pair $\langle\mathcal{F} o h n, \mathcal{F} o b n\rangle$ : the property of the subject pair 〈John, John $\rangle$ being a shaving (i.e. shaver-shaved) pair. More generally, repeated variables have to be handled by means of ordered sets: $R x x \equiv\langle x, x\rangle \in\{\langle y, z\rangle \mid R y z\}$. Forgetting this, the received view of reflexives, within the Fregean tradition, has been that they turn binary relations into unary properties by identifying their two argument positions. But that there is something amiss with this line of analysis is very evident, since, notoriously, there is a severe problem with anything of the form

$$
x \in\{y \mid P y\} \equiv x \notin x,
$$

in which there is taken to be a unary property, while there is no corresponding problem with

$$
\langle x, x\rangle \in\{\langle y, z\rangle \mid y \notin z\} \equiv x \notin x,
$$

in which a binary relation is involved. Substituting the given set-abstraction expression for ' $x$ ' in the former produces the well-known contradiction

$$
\{y \mid P y\} \in\{y \mid P y\} \equiv\{y \mid P y\} \notin\{y \mid P y\},
$$

but the corresponding substitution with the latter produces merely

$$
\begin{aligned}
\langle\{\langle y, z\rangle \mid y \notin z\},\{\langle y, z\rangle \mid y \notin z\}\rangle \in\{\langle y, z\rangle \mid y \notin z\} \equiv \\
\{\langle y, z\rangle \mid y \notin z\} \notin\{\langle y, z\rangle \mid y \notin z\},
\end{aligned}
$$

i. e. something of the form

$$
\langle a, a\rangle \in a \equiv a \notin a,
$$

which is not contradictory. So this relation between $x$ and itself provably cannot be configured as a unary property of $x$, and it cannot be presumed, as a result, that abstracting 'John' entirely from 'John shaves John' leaves a predicate just of 'John'. Abstraction separately, i. e. with distinct variables, from any or all of the individual subject places in an elementary sentence is entirely possible, but repetition of the same individual subject must respect the undoubted fact that $\neg(\forall R)(\exists P)(R x x \equiv P x)$. That is why ' $\lambda x(x \text { is not a member of } x)^{\prime}$ does not denote a property: because, in the first place, ' $x$ is not a member of $x$ ' is not a predicate with a single subject ' $x$ ', but instead a relation-the relation 'is not a member of'-with subjects ' $x$ ' and ' $x$ '. So the relation must be identified independently of the repetition of ' $x$ ', and have a pair of argument places: $R x x \equiv \lambda y \lambda z \operatorname{Ryz}(x)(x) .1$

\section{REFERENCES}

'The author would like to thank two anonymous referees for the $A \mathcal{F} L$, whose promptings improved this paper considerably. 
Cocchiarella, N. 1986, Logical Investigations of Predication Theory and the Problem of Universals, Bibliopolis, Naples.

Cocchiarella, N. 1987, Logical Studies in Early Analytic Philosophy, Ohio State University Press, Columbus.

Geach, P. T. 1972, Logic Matters, Blackwell, Oxford.

Geach, P. T. and Black, M. (editors) 1952, Translations from the Philosophical Writings of Gottlob Frege, Blackwell, Oxford.

Goodstein, R. L. 1958, 'On the Formalisation of Indirect Discourse', fournal of Symbolic Logic 23, 417-419.

Haack, S. 1978, Philosophy of Logics, Cambridge University Press, Cambridge.

Kneale, W. 1972, 'Propositions and Truth in Natural Languages', Mind 8I, 225-243.

Kneale, W. and Kneale, M. 1962, The Development of Logic, Clarendon, Oxford.

Leisenring, A. C. 1969, Mathematical Logic and Hilbert's \&-Symbol, Macdonald, London.

Prior, A. N. 1971, Objects of Thought, Oxford University Press, Oxford.

Slater, B. H. 1986, 'E-type Pronouns and $\varepsilon$-terms' Canadian fournal of Philosophy I6, 27-38.

Slater, B. H. 2000, 'Concept and Object in Frege', Minerva Volume 4 (http://www.ul.ie/ philos/vol4/index.html)

Slater, B. H. 200I,'Prior's Analytic Revised', Analysis 6I, 65-8I.

Slater, B. H. 2002, 'Syntactic Liars', Analysis 62, 107-109. 
The Australasian fournal of Logic (ISSN I448-5052) disseminates articles that significantly advance the study of logic, in its mathematical, philosophical or computational guises. The scope of the journal includes all areas of logic, both pure and applied to topics in philosophy, mathematics, computation, linguistics and the other sciences.

Articles appearing in the journal have been carefully and critically refereed under the responsibility of members of the Editorial Board. Only papers judged to be both significant and excellent are accepted for publication.

The journal is freely available at the journal website at

http://www .philosophy . unimelb.edu.au/ajl/

All issues of the journal are archived electronically at the journal website.

SUBSCRIPTIONS Individuals may subscribe to the journal by sending an email, including a full name, an institutional affiliation and an email address to the managing editor at ajl-editors@unimelb.edu.au Subscribers will receive email abstracts of accepted papers to an address of their choice. For institutional subscription, please email the managing editor at ajl-editors@unimelb.edu.au.

Complete published papers may be downloaded at the journal's website at http://www.philosophy.unimelb.edu.au/ajl/ The journal currently publishes in pdf format.

Submission The journal accepts submissions of papers electronically. To submit an article for publication, send the ${ }^{\Delta} \mathrm{T}_{\mathrm{E}} \mathrm{X}$ source of a submission to a member of the editorial board. For a current list of the editorial board, consult the website.

The copyright of each article remains with the author or authors of that article. 